\section{§2. Subcooled He II Heat Transport in the Channel with Abrupt Contractions/Enlargements}

Maekawa, R.

Subcooled superfluid helium (He II) has been widely used as a coolant for large scale superconducting magnets because of its excellent heat transport capability under steady state and transient conditions. Since the operating temperature of superconducting magnet becomes $1.9 \mathrm{~K}$ or so, the critical current and magnetic field also increases. Generally, the superconducting magnet which is operated with $\mathrm{He}$ II shows higher stability margin than that of normal helium (He I). However, the design of superconducting magnet has some crucial points such as, geometry of cooling channels, availability of He II adjacent to the conductor and so on.

Helical coil system of the LHD will be cooled with subcooled He II for the Phase II operation so that the heat transport mechanisms of He II require intensive research for the successful LHD upgrade. To study the heat transport characteristics within the windings, simple channels were designed.

The channel, made of G-10, contains contraction geometry to simulate the cooling channel of superconducting coils as shown in Fig. 1. A heater was embedded in the copper block which provide heat input to the channel from one end, while the other end is open to the He II bath. Seven thermometers were located within the channel to measure temperature gradients in He II. Samples are installed in the He II cryostat as shown in Fig. 2.
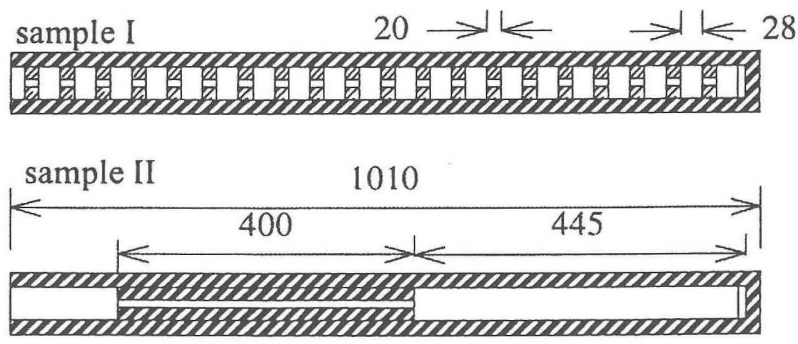

Fig. 1. Schematic of two channel samples; sample I with one contraction, sample II with evenly spaced contractions.

Measurements were conducted with three different bath temperatures $1.83,1.96$ and $2.05 \mathrm{~K}$. Fig. 3 shows the experimental results with theoretical calculation. Calculation was performed, solving one-dimensional turbulent heat transport equation;

$$
Q^{3}=\frac{1}{g_{e q}^{3}} \int_{T_{b}}^{T_{\max }} \frac{d T}{f(T)}
$$

where $g_{\text {eq }}$ is equivalent geometry function[1], $f(T)$ is heat conductivity function for He II[2].

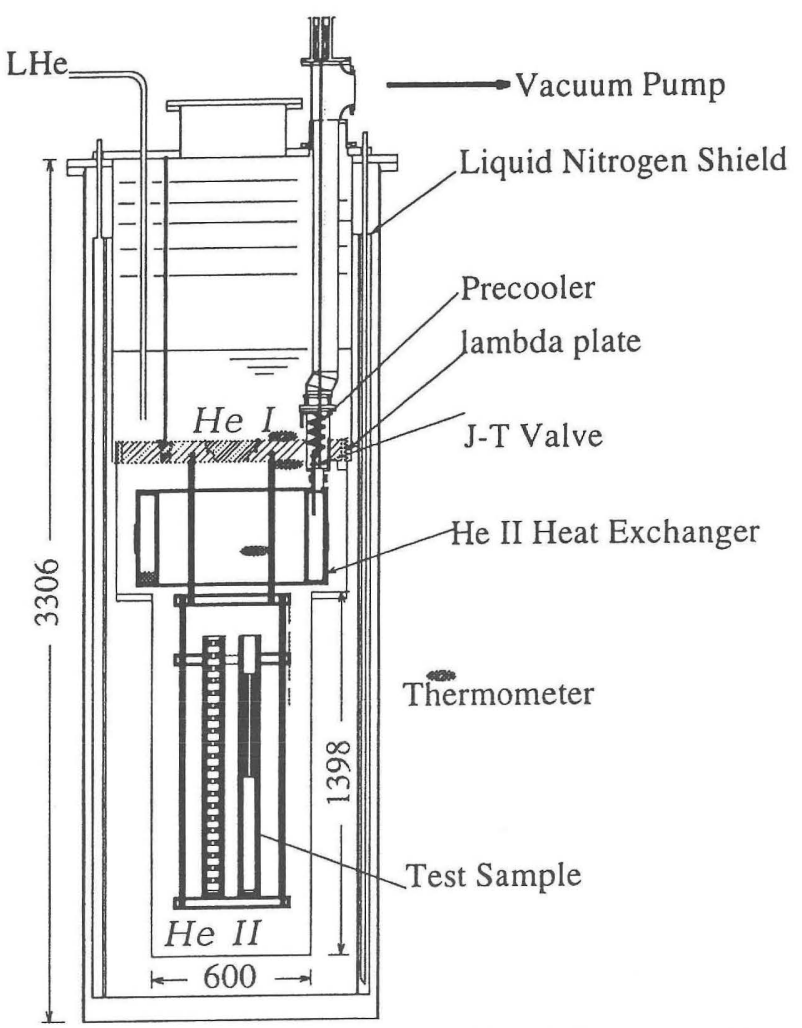

Fig. 2. Schematic of Subcooled He II Cryostat with test sample.

Results indicate that the channel with evenly spaced contractions/enlargements, sample II, has lower effective heat transport area than that of one contraction channel (sample I). This is qualitatively explained since the heat stream of He II is more affected with entrance/exit region so that the heat transport capability is more affected with the numbered channels placed inside the samples.

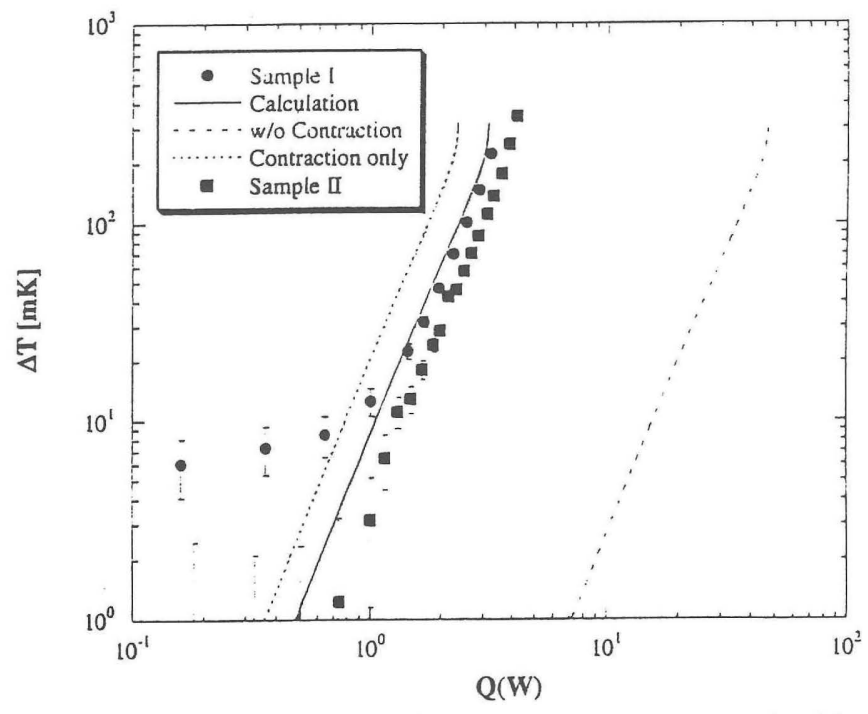

Fig. 3. Heat transport experimental results compared with one-dimensional heat transport equations at $\mathrm{T}_{\mathrm{b}}=1.83 \mathrm{~K}$

Reference

1) Pfotenhauer, J.M. Cryogenics 32, (1992) 466

2) Van Sciver, S.W. Helium Cryogenics Plenum Press. 